This is an electronic reprint of the original article. This reprint may differ from the original in pagination and typographic detail.

Author(s): Jantunen, Tommi

Title: Clausal coordination in Finnish Sign Language

Year: $\quad 2016$

Version:

Please cite the original version:

Jantunen, T. (2016). Clausal coordination in Finnish Sign Language. Studies in Language, 40(1), 204-234. https://doi.org/10.1075/sl.40.1.07jan

All material supplied via JYX is protected by copyright and other intellectual property rights, and duplication or sale of all or part of any of the repository collections is not permitted, except that material may be duplicated by you for your research use or educational purposes in electronic or print form. You must obtain permission for any other use. Electronic or print copies may not be offered, whether for sale or otherwise to anyone who is not an authorised user. 
When citing, please use the published article: Jantunen, Tommi (2016). Clausal coordination in Finnish Sign Language. Studies in Language 40:1, 204-234. DOI: 10.1075/sl.40.1.07jan. The published article is under copyright and the publisher should be contacted for permission to re-use or reprint the material in any form.

\title{
CLAUSAL COORDINATION IN FINNISH SIGN LANGUAGE
}

Tommi Jantunen, University of Jyväskylä, tommi.j.jantunen@jyu.fi

\section{Author's contact information:}

\author{
Tommi Jantunen \\ University of Jyvaskyla \\ Department of Languages \\ Sign Language Centre \\ P.O. Box 35 \\ FI-40014 University of Jyvaskyla \\ Finland
}

E-mail: tommi.j.jantunen@jyu.fi 
Abstract

3 This paper deals with the coordination of clauses in Finnish Sign Language (FinSL).

4 Building on conversational data, the paper first shows that linking in conjunctive

5 coordination in FinSL is primarily asyndetic, whereas in adversative and disjunctive

6 coordination FinSL prefers syndetic linking. Secondly, the paper investigates the

7 nonmanual prosody of coordination: nonmanual activity is shown both to mark the

8 juncture of the coordinand clauses and to draw their contours. Finally, the paper

9 addresses certain forms of clausal coordination in FinSL that are sign language-

10 specific. It is suggested that the sign language-specific properties of coordination are

11 caused both by the fact that signers can use two manual articulators in the production

12 of sentences, and by the pervasive iconicity of sign language structure.

13 Key words: coordination, clause, prosody, nonmanual element, modality-difference,

$14 \quad$ Finnish Sign Language

15

\section{Introduction}

18 This paper deals with clausal coordination - the linking of two (or more) clauses of

19 the same rank (Haspelmath 2007) - in the majority sign language of Finland, Finnish

20 Sign Language (FinSL). FinSL has approximately 3000 native users, in addition to

21 which circa 6000-9000 people use FinSL as a second language (Takkinen et al.

22 forthcoming). There is also a small minority sign language in Finland, Finland-

23 Swedish Sign Language (FinSSL). FinSSL is used in the coastal areas of Finland by

24 circa 150 people with mainly Swedish-speaking family and school backgrounds

25 (Hoyer 2012). Historically, both FinSL and FinSSL are related to Swedish Sign 
26 Language (SSL), which was brought to Finland in the middle of the 19th century by

27 the founder of Finnish Deaf education, Carl Oscar Malm. Today FinSL and SSL are

28 not mutually intelligible, but due to family background and language contacts,

29 FinSSL and SSL users can in fact understand each other rather easily (Hoyer 2012).

30 Complex sentences of any kind have not been previously investigated in FinSL,

31 in which prior syntactic work has been done on simple equative sentences (Jantunen

32 2007), transitive clauses (Jantunen 2008), topic-comment structures (Jantunen 2009),

33 and clause-internal ellipsis (Jantunen 2013). However, internationally, issues relating

34 to complex sentences in sign languages have been addressed at least since Liddell

35 (1980), and the most recent overviews on the subject are Tang and Lau (2012) and

36 Velupillai (2012: 339-342). A rather salient feature of the international research

37 conducted so far on the complex sentences of sign languages is that it has focused

38 mainly on subordinate structures, particularly on relative clauses. Explicit work on

39 coordinate structures has been relatively marginal, a recent example being Davidson

40 (2013) for American Sign Language (ASL) (see also, for example, Johnston \&

41 Schembri 2007 and Hodge \& Johnston 2013 for Australian Sign Language, Auslan).

\subsection{Theoretical background and the organization of the paper}

45 As there has been no previous work on clausal coordination in FinSL, this paper first

46 discusses (Section 4) the basic structure of the three most recognized types of

47 coordinated clauses: conjunctively ('and'), adversatively ('but'), and disjunctively

48 ('or') coordinated clauses (Haspelmath 2004, 2007). The prototypes of these

49 constructions are highly symmetric, that is, the clauses combined are, in the standard

50 case, both autonomous and isolated. Consequently, the main discussion on coordinate 
51 clauses concerns the explicitness of their linkage. In traditional terms, this

52 corresponds to answering the question whether the coordination is manifested

53 syndetically (i.e. with a coordinator) or asyndetically (i.e. without a coordinator)

54 (Gast \& Diessel 2012).

55 Work on clause linkage has proposed that coordination may be considered to manifest itself also asymmetrically, that is, so that only one of the coordinands can 57 stand alone as an independent unit (e.g. Bril 2010; Reintges 2010). On the level of 58 clauses, the notion of asymmetric coordination refers, in practice, to the clause chain construction found, for instance, in the languages of Papua New Guinea, Australia, and the Americas (Velupillai 2012). In clause chaining, typically more than two

61 clauses are combined without any overt syntactic marker, and typically only the last 62 clause in the string is inflected for TMA (Velupillai 2012). However, clause chaining 63 as an instance of asymmetric coordination is still the subject of some theoretical 64 debate (Van Valin 2005; Bickel 2010; Foley 2010; Gast \& Diessel 2012), mainly because the phenomenon can also be seen as a manifestation of cosubordination, an intermediate linking strategy between coordination and subordination (Van Valin \& LaPolla 1997; Butler 2003; Van Valin 2005; Velupillai 2012; Gast \& Diessel 2012), or even as a complementation strategy, an alternative way of building subordinatelike complement constructions (e.g. Dixon 2006). Due to this theoretical ambiguity, and because the present work is the first attempt to address coordination in FinSL, possible clause chains and, more generally, also other forms of asymmetric coordination will not be explicitly discussed in the present paper. Concerning sign languages, nonmanual prosody - the features in signing manifested by the activity of the body, head, and the parts of the face (Sandler 2012) - has been argued to play an important role in the formation of complex sentences. 
Most notably, it has been argued that nonmanual prosody marks especially the boundary between linked clauses (e.g. Tang \& Lau 2012). However, the exact nature of this marking in coordination is not fully known and will be discussed for FinSL in Section 5. The discussion in this section will also address the role that nonmanual elements - the head in particular - have in marking the prosodic contours, the domains from the perspective of their shape, of coordinated clauses.

The present study operates on the principle that the core phenomena concerning clausal coordination in FinSL can be described and analyzed using general notions developed and employed in the analysis of spoken languages (for a slightly alternative view, see Hodge \& Johnston 2013). However, sign languages are different from spoken languages in terms of their physical manifestation and this fact brings up, by necessity, certain modality differences between the two types of languages. Some of these will be addressed in Section 6 from the perspective of coordination in FinSL. In general, we believe that the discussion of coordinate constructions that are not detectable on the basis of research into spoken languages only adds positively to our understanding of the cross-linguistic nature of clausal coordination.

In terms of the general theoretical underpinning, the present study is tied, like previous work on FinSL syntax, to the typologically motivated functional framework. More specifically, the study is grounded on the principles of the Basic Linguistic Theory (Dryer 2001, 2006; Dixon 2010), which is an informal (as opposed to formal) descriptive framework widely used by linguists to describe and compare the grammars of individual languages. Concerning typological theory in particular, the study approaches clausal coordination from a superficially non-parametric, traditional perspective (e.g. Haspelmath 2004, 2007; Velupillai 2012). However, this is not to say that recent advances in parameter-based approaches to clause-linking have been 
101 neglected. On the contrary, the influence of the parameter-approach on the present

102 study is seen most clearly in the way the whole notion of clausal coordination is

103 conceptualized: the paper assumes that clausal coordination is not a holistic and easily

104 categorizable phenomenon, but a gradual one (Gast \& Diessel 2012).

\subsection{On the data and methodology}

The present study uses the widest collection of annotated video material and other data so far available for FinSL. The main video material comprises circa 45 minutes

111 FinSL signers (2 male, 3 female; ages between 20 and 60 years). The material was

112 recorded in a studio with multiple cameras and in a dialogue-like setting in which

113 each signer either told the other signer a story about a cartoon (The Snowman, or

114 Frog, Where Are You?), or told the other signer about his/her hobby or other interests

115 (e.g. ice hockey, languages, their studies etc.) (for more details, see Jantunen et al.

1162012 and Puupponen et al. 2014). All of the material has been appended with

117 annotations for signs and with sentence-level translations in ELAN (Crasborn \&

118 Sloetjes 2008). In order to identify instances of clausal coordination, a team of

119 researchers went through the material several times and picked out a representative

120 selection of coordinate examples $(n=50)$. These examples were then discussed for

121 their grammaticality and variability with native signers. However, no systematic

122 elicitation was conducted.

123 An important feature of the main video material is that numerical data on the

124 head movements of the signers has been added to a 40 minute stretch of it. This has

125 been achieved with the help of computer-vision technology, implemented in the 
126 SLMotion software specifically developed for the purpose of analyzing the motion of 127 different articulators in sign language videos (Karppa et. al 2014). In particular, the 128 computer vision technology was used to calculate the yaw (the turning movements of 129 the head), pitch (the nodding movements of the head), and roll (the tilting movements 130 of the head) angles of the heads of the signers appearing on the video (Luzardo et al. 131 2013). The same technology has also been used to estimate the amount of horizontal 132 (x-axis) and vertical (y-axis) motion of the centroids of the signers' heads.

133 The computer-vision based data on head movements is used to support the 134 investigation of the prosody of coordinated clauses in FinSL (Section 5). To 135 strengthen the analysis of nonmanual prosody even further, the computer-vision 136 processed material has also been annotated for linguistically significant head 137 movements by an experienced research assistant. The types of head movement 138 identified in this task were the nod, thrust, pull, turn, and tilt together, with their 139 reduced (cf. chin-up, chin-down) and repetitive variants (cf. nodding, headshake, 140 tilting).

141 Concerning the data, two notes are in order. First, a small portion of the main 142 video material (ca. 5 minutes) was recorded with Motion Capture (mocap) technology 143 (Jantunen et al. 2012). However, although some examples and figures in this paper 144 show mocap related labels and equipment, the numerical mocap data has not been 145 exploited in the present study. Second, due to its relatively small quantity, all of the 146 data in this paper (also the computer-vision based SLMotion data) has been 147 approached qualitatively. Consequently, the present study does not include, for 148 example, any statistical or number-based generalizations. 

in Section 4. Before that, Sections 2 and 3 introduce the notion of clausal coordination and some basics of FinSL syntax in more detail.

152

\section{Clausal coordination from the typological perspective}

\subsection{The basics of clausal coordination}

156

157 Haspelmath (2007: 1) defines coordination generally as a term that "refers to

158 syntactic constructions in which two or more units of the same type are combined into

159 a larger unit and still have the same semantic relations with other surrounding

160 elements." He continues that these units - coordinands - may be words (e.g. verbs),

161 phrases (e.g. noun phrases) or full clauses or sentences. In this paper, coordination is

162 understood in the sense of Haspelmath and discussed explicitly only on the sentential

163 level.

164 The three main semantically based types of coordination found in the world's

165 languages are conjunctive ('and'; cf. the semantic notion of adding), disjunctive ('or';

166 cf. the semantic notion of alternation) and adversative ('but'; cf. the semantic notion of

167 contrast) coordination (Haspelmath 2004, 2007; Velupillai 2012). These are

168 exemplified for English in (1a), (1b) and (1c), respectively.

169

170

(1) a. [John likes Mary] and [Mary likes John].

b. [Should John stay at home] or [should he go to Mary's]?

172

c. [Mary went to a store] but [John stayed at home]. 

reason) as a major semantically based type of coordination (e.g. Mary was unhappy to see that it was raining for she had no umbrella with her). However, cross-

177 linguistically, causal coordination is not as fixed a coordination type as the other three 178 (Haspelmath 2004, 2007; Velupillai 2012) and it is thus not discussed in the present 179 paper.

Formally, as introduced in Section 1, coordination may be asyndetic or syndetic 181 (Haspelmath 2004, 2007; Velupillai 2012). In asyndetic coordination there is no overt 182 linker and the coordinands are simply juxtaposed (cf. A B). Syndetic coordination, on the other hand, involves an overt linking device (coordinator, coordinating conjunction), such as the and, or, and but found in Example (1). Cross-linguistically, 185 the coordinator may either precede the unit it is coordinating (e.g. A and B), or follow 186 it (e.g. A B and). In the former case, the coordinator is labeled prepositive while in 187 the latter case it is called postpositive. The coordinators may be free words or bound 188 clitics.

189 In syndetic coordination, there may be either one (e.g. A and B) or two 190 coordinators (e.g. and A and B), that is, the coordination may be either monosyndetic 191 or bisyndetic. In monosyndetic coordination, distinguishing between prepositive and 192 postpositive coordination may not be straightforward (i.e. is it A and B or A and B). 193 Clisis and prosodic information are typically used as evidence (Haspelmath 2004). 194 More than two units can also be coordinated and this is referred to as multiple 195 coordination. In multiple coordination, many languages allow coordinator omission, 196 but often the last coordinator has to be expressed. Overtly expressing the coordinator 197 in other cases may indicate emphasis or be otherwise overtly marked (Haspelmath 198 2004; Velupillai 2012). 
zero anaphora, in which the referent mentioned in the first clause is referred back to

201 by means of zero in the second clause. Languages differ in the degree to which they

202 allow zero anaphora. In English, for example, the phenomenon is restricted in such a

203 way that it is possible to say The man came in and saw the woman but not The man

204 came in and the woman saw (i.e. English has an S/A pivot constraint). In Dyirbal,

205 however, the latter sentence is accepted but not the former (i.e. Dyirbal has an S/P

206 pivot) (Dixon 1994). On the other hand, in Mandarin Chinese, it is possible to use

207 both types of sentences (i.e. there is no pivot constraint), as the primary recovery

208 method of the unexpressed argument in Mandarin is, ultimately, world and contextual

209 knowledge (see Huang 2000).

\subsection{Remarks on clausal coordination in sign languages}

212

213 According to Velupillai (2012), coordination in sign languages tends to be asyndetic,

214 that is, the coordinated units are typically simply juxtaposed without an overt linker.

215 However, some syndetic coordinated constructions can be found. For example, ASL

216 has at least two lexical items (fingerspelled o-r and the sign OR-WHICH) for the

217 expression of disjunctive coordination and, in addition to these, ASL also has a

218 general use coordinator COORD that conveys both disjunctive and conjunctive

219 linking (Davidson 2013) (for the notational conventions of sign language examples,

220 see Appendix 1; unless otherwise explicitly stated, all of the examples in the paper

221 follow these guidelines). On the other hand, in SSL, the sign PLUS (cf. Figure 2 in

222 Section 4) can be used to coordinate units conjunctively, although in general SSL

223 prefers asyndetic conjunctive linking. Overall, there seems to be a tendency that if a 
sign language uses conjunctions, they occur most often in disjunctive and in

225 adversative coordination. Example (2) demonstrates syndetic adversative coordination

226 with the sign BUT in Auslan (Johnston \& Schembri 2007: 213; original notation).

227

228

229

230

231

232

233

234

\section{(2) k-i-m LIKE CAT BUT p-a-t PREFER DOG}

'Kim likes cats but Pat prefers dogs.'

No properly supported claims have been made as to whether syndetic

coordination in sign languages is prepositive or postpositive, or (primarily)

monosyndetic or bisyndetic. However, the data found in the literature seems to speak for prepositive monosyndetic coordination.

Multiple coordination is possible also in sign languages and it is primarily asyndetic. However, according to Velupillai (2012), multiple items can also be coordinated in a syndetic fashion with the so-called list buoy construction (Liddell 2003). In the list buoy construction, the fingers of the non-dominant hand serve as the "numerical base" to which the index finger of the dominant hand points in between the coordinated items/events. As each of the coordinands is preceded by a numerical index, the list buoy strategy can be treated as a sign language-specific example of prepositive (bi- or) multisyndetic coordination (cf. first A, second B, third C). An example of a non-coordinatively used list buoy from FinSL is given in Figure 1.

\section{\{Figure 1 approximately here.\}}

$$
\text { An important feature of clause linking in sign languages is the use of non- }
$$
manual activity, usually equated with prosody (e.g. Sandler 2012; for nonmanuality in 
general, see Pfau \& Quer 2010). Concerning coordination, Davidson (2013), for example, has described how items in ASL may be coordinated disjunctively and conjunctively only by shifting the body slightly for each coordinated element, and

252 thus signing each of the coordinated items in a separate location in the signing space.

253 On the other hand, Tang and Lau (2012) have argued that nonmanuality is used to

254 mark especially the juncture of the coordinated clauses: according to them, the

255 boundary between linked clauses is often the domain of an extended head nod or a 256 body turn/position change, for instance, in Hong Kong Sign Language (HKSL) and in 257 ASL.

258 In general, it has been argued that the significance of nonmanual 259 activity/prosody is higher if manual markers of coordination are absent (Tang \& Lau 260 2012). Interestingly, however, although an important role has been attributed to 261 nonmanuality in clause linking in sign languages, no in-depth investigations of its role 262 in clausal coordination exist in the literature.

\section{3. Background on simple sentences in FinSL}

\subsection{Word classes}

268 On a general level, the FinSL lexicon contains four types of signs: word-like signs, 269 signs including gestural components (in the sense of Liddell 2003), emblems, and 270 pantomimic gestures. Of these, the first two can be further divided into word classes

271 while the latter two are types of gestures (Jantunen 2010). The major word classes in

272 FinSL are nominals and verbals, defined by semantic and grammatical criteria. The 273 adjective is not treated as an independent word class in FinSL research. Signs 
274 denoting property are analyzed semantically and grammatically either as marginal

275 nominals or marginal verbals (Jantunen 2010).

276 The typology of FinSL verbals is relevant in a discussion of clausal structure

277 and coordination. In general, FinSL verbals are analyzed as belonging to one of three

278 main subcategories: Type 1, Type 2, and Type 3 verbals (Jantunen 2008, 2010,

279 2013; see also Rissanen 1998, and Liddell 2003). Type 1 verbals consist of only a

280 morphological component and are formationally the most fixed type of FinSL verbals.

281 Type 2 verbals include both a morphological and a gestural component, the latter of

282 which allows the verbals to be directed meaningfully in space in order to indicate, for

283 example, discourse participants. Morphological and gestural components are present

284 also in Type 3 verbals. In these verbals, the morphological component is a classifier

285 handshape morpheme while the gestural component functions to depict mainly

286 topographic locations.

\subsection{The structure of simple sentences}

FinSL (declarative) clauses with a Type 1 or Type 2 verbal predicate are organized in such a way that the most important core argument always comes before the verbal

292 (V). With respect to intransitive clauses, this yields the basic clausal structure SV.

293 For transitive clauses, this produces the basic clausal structures AVP and APV

294 (Jantunen 2008).

The syntactic status and behavior of Type 3 verbals are different from those of

296 Type 1 and 2 verbals. First, Type 3 verbals are considered to be full, well formed

297 clauses on their own (Jantunen 2008, 2013). This analysis stems partly from the fact

298 that these verbals include a fused classifier handshape or handshapes that can be 
analyzed as nominal core arguments of the predicate (Van Valin \& LaPolla 1997;

Dixon \& Aikhenvald 2000; Van Valin 2005). Another reason for the clausal interpretation is the fact that the meaning of these verbals is typically very clause-like, that is, it covers the whole event. Due to the semantics of classifiers, however, there is often some vagueness in the meaning of such verbals/clauses.

304 Second, as Type 3 verbals are inherently fully fledged clauses, they are typically

305 involved in constructions that rank above the level of the clause; in practice, this

306 means in FinSL the topic-comment structure. In these structures, Type 3

307 verbals/clauses are always (the final part of) the comment, expressing the main

308 predication. Topics of topic-comment structures are clause-external, prosodically left-

309 detached NPs whose function is to set an interpretative framework for the comment.

310 Formationally, topics in FinSL are marked nonmanually (the eyes are widened and

311 eyebrows are raised). The structural schema of topic-comment structures is $\mathrm{TOP}_{\mathrm{NP}}+$ $312 \mathrm{COM}_{\text {clause }}$

313 Clauses with a nominal predicate (e.g. identifying clauses, various

314 characterizing, locative etc. clauses) are typically formed by simply juxtaposing two 315 NPs (Jantunen 2007, 2013). In other words, FinSL does not have a copula between 316 the (first) non-predicating and the (second) predicating NP. Often identifications and 317 predications about location etc. are expressed with the topic-comment structure. One 318 practical reason for this is that this structure makes the syntactic inter-connectedness 319 of the NPs more salient than a simple clause-internal parataxis of the elements.

320 In FinSL, the basic means of making a declarative sentence negative or 321 interrogative is by adding to it a nonmanual operator. The negative operator in 322 FinSL is a headshake (Rissanen 1985), the scope of which is the whole sentence 323 (excluding the possible topic). The nonmanual negative operator can be accompanied 
324 by manual negative signs, but there is no single manual negator in FinSL (Zeshan

325 2004). The interrogative operator in FinSL has two main forms, one for polar

326 (yes/no) questions and the other for content (wh-) questions. In the main polar

327 question marker, the eyebrows are raised and the head tilts forward. In the main

328 content question marker, the brow is furrowed and the head tilts forward. The scope

329 of the nonmanual interrogative operator is conditioned similarly to that of the negative

330 operator (Rissanen 1985; Savolainen 2006). Question words are used with content

331 questions and they can occur in various positions, but the clause-initial (after the

332 topic) and clause-final positions are the most typical.

333

334

\subsection{Clauses in discourse}

The appearance of FinSL clauses and sentences in natural discourse is somewhat different from their appearance in isolated examples (Jantunen 2008). The differences lie mainly in the meaningful use of signing space, blending, and frequent ellipsis. As these phenomena are important for the understanding of FinSL examples in the following sections, they are briefly illustrated here with the following short text from

341 Suvi, the Online Dictionary of FinSL (article 4, example 2):

(3) line 1: COMPUTER / ME blend:(TO-TYPE-KEYBOARD-2 ${ }_{\text {low }}$ ) /

line 2: ME blend:(TO-LOOK-AT- $2_{\text {up }}-2_{\text {down }} /$

line 3: polar:[BETTER both_hands $C L-C-"$ change-places"- $\left.\left.2_{\text {up }} \mid 2_{\text {down }}\right]\right) /$

line 4: ME blend:(TO-PUNCH-KEYBOARD-2 ${ }_{\text {low }} /$

line 5: both_hands $C L-C-"$ change-places"- $2_{\text {up }} \mid 2_{\text {down }} /$

line 6: FINE) 
'I was typing with the computer. When I was reading the text I started to wonder whether the order of the two paragraphs should be changed. I typed in the command and the order changed. Now the text was fine.' on its own line. The sentence in line 1 is a topic-comment structure in which the sign COMPUTER is the topic and the subsequent SV-ordered intransitive clause is the comment. The sentence in line 2 is a structurally incomplete AV-ordered transitive clause in which the P-argument (cf. 'screen' or 'text') is not expressed lexically. The sentence in line 3 is an interrogative intransitive sentence (i.e. a polar question); it is an example of a type of intransitive structure in which the precise meaning of the fused classificatory core argument of the Type 3 verbal is inferred only from the context. The sentence in line 4 is a minimal structurally full intransitive clause with the order SV. The sentence in line 5 is also a minimal intransitive clause, this time, however, expressed by a single Type 3 verbal. The sentence in line 6 is a nominal

364 characterizing clause with an omitted clause-initial thematic NP. examples. The first concerns the production of the signs TO-TYPE-KEYBOARD (line 1) and TO-PUNCH-KEYBOARD (line 4) and the second concerns the

369 (lines 3 and 5). In both cases, the signs involved are produced in iconically motivated 370 locations, of which one (indicated with the index $2_{\text {low }}$ ) corresponds to the assumed 371 location of a computer keyboard (in front of the signer, low) and the other (indicated 372 with the index 2) the assumed location of the computer screen (in front of the signer, 
373 on the level of the eyes). These locations remain the same throughout the text, which

374 indicates that locations of space are used as a reference-tracking device.

375 Concerning blends - the pantomimic aspects of signing whose semantic

376 deciphering requires the mental construction of meaning (see Liddell 2003) - the text

377 in (3) contains several examples as well. For example, it is not the clause in line 2, or

378 any other syntactic unit (in the traditional sense) directly linked to it, which expresses

379 the thematic information 'text or paragraphs on the computer screen'; the P-argument

380 that could be supposed to express this meaning is omitted from the clause. Instead, the

381 meaning is constructed mentally on the basis of both the text-initial topic 'computer' -

382 limiting the typing process to the domain of computers and not, for example, to the

383 domain of typewriters - and the blend in which the signer imagines and, accordingly,

384 pantomimically creates the computer screen in front of her. Blends are also an

385 important means of indicating and increasing textual cohesion. For example, the final

386 nominal clause in line 6 does not need any additional lexical or morphosyntactic

387 material to express the information that it is the 'text' or 'paragraphs' that are fine,

388 because the continuity of the theme expressed by the clause in line 5 is made explicit

389 by the continuity of the blend. In other words, it is the blend that ties the themes of the

390 two clauses together.

391 The use of space and blending contribute positively to the construction of

392 meaning, so that a lot of syntactically important material can be left out of FinSL

393 sentences. For example, in (3), only two (in lines 1 and 4) of the total of six clauses

394 can be analyzed as being syntactically full. It has been argued that this type of

395 frequent ellipsis - the optional non-expression of syntactic material (McShane 2005)

396 - is very frequent in FinSL. For example, Ala-Sippola (2012) calculates that over half

397 of transitive clauses in her data were produced elliptically without their S/A argument. 
According to Jantunen (2013), FinSL tends to elide especially the thematic nominal

399 elements, and this is the case also in (3). The frequent omission of thematic elements

400 has been used as evidence for the interpretation that FinSL is inherently a discourse-

401 oriented language (Jantunen 2013). In such languages, ellipsis ultimately serves to

402 add discourse coherence (McShane 2005).

403 Concerning the elliptical process of zero anaphora, introduced in Section 2.1,

404 FinSL behaves similarly to Mandarin. In other words, FinSL has no S/A or S/P pivot

405 that could be referred to in the discussion of clausal coordination (Jantunen 2013).

406

407

\section{Coordinating clauses in FinSL}

408

409

\subsection{Conjunctive, adversative, and disjunctive coordination}

411 The data from FinSL (see Section 1.2) shows that, like other sign languages

412 researched for coordination (see Section 2.2), also FinSL can conjoin clauses by

413 simply signing them one after the other. Let us begin by considering Examples (4)414 (6).

(4) BOY LOOK-AT ' REALLY NOTIFY DOG PALM-UP / PALM-DOWN blend:([SEARCH:durative] ' [DOG HELP SEARCH STUDY]) 'The boy looked at the jar and then turned to the dog like "what now". Well, the boy looked for the frog intensively and the dog helped him in the search.' 


\section{(5) [HOME+GO] ' [INDEX SNOWMAN GO]}

'They went home and the snowman left.'

(cfins12013-005-05, signer 1, coo-7)

\section{(6) [BE-PRESENT HELSINKI] ' [RETURN RECENTLY]}

'We were there in Helsinki and came back only recently.'

(mocap data 2, signer 1, example 21)

The sentences marked in square brackets in (4), (5), and (6) all represent conjunctive coordination of two FinSL clauses (of which at least one has always undergone ellipsis of a thematic element; the sentence in 4 also involves the blending of the signer into the boy). None of the examples involve any overt linking device in between the clauses and, according to the data and the intuitions of native informants, this is indeed the most typical way to express conjunctive coordination in FinSL. What is always present in the construction is the prosodic break between the clauses. In (4)-(6), this break manifests most notably as a change in the head and body position (see Section 5 for more discussion).

In FinSL - as, for example, in ASL and in SSL (see Section 2.2) - it is possible to express conjunctive coordination also with a syndetic strategy. In such cases, the primary coordinators are the sign ALSO and (as in SSL) the sign PLUS, both used in between the coordinated clauses (see Figure 2). One should note, however, that in conjunctive coordination the syndetic strategy is always a marked option. This is reflected also by the fact that the signs ALSO and PLUS have other, non-coordinative functions in FinSL (cf. the meaning 'too' of the sign ALSO; the sign PLUS, on the other hand, is used primarily in mathematical settings). 
451 "hooking" c-shaped hands together in the vertical dimension. However, the sign AND

452 belongs to the register very close to Finnish and is therefore not used by native FinSL

453 signers in everyday conversation.

454 Other semantically based types of coordination are also found in FinSL. The

455 following are examples of adversative clausal coordination:

456

(7) $\quad$...[NICE] ' [TIRED EXHAUSTED]

(The boy was happy and he waved farewell to the snowman.) He had had fun but now he was totally exhausted.

(8) ...MAYBE STILL PRACTICE ICE-HOCKEY SURE INDEX ' BUT NEVERTHELESS FAVOR DECREASE '...people still practice ice hockey but its popularity has diminished.' (cfinsl2013-008-06, signer 1, coo-4)

(9) HELP YES ' BUT NOW INDEX NO-MORE ZERO

'It helped at the time but now I don't have an interpreter with me anymore.'

471 linker and with a break in prosody between the clauses, just like in conjunctive 
472

473

474

475

476

477

478

479

480

481

482

483

484

485

486

487

488

489

490

491

492

493

494

495 coordination (for more, see Section 5). In (8) and (9), in addition to a break in prosody, there is also a linking sign BUT between the clauses (see Figure 2). On the basis of the present data and native intuitions, the use of this sign is very common in adversative coordination in FinSL, and it can also be inserted at the clausal juncture of the example in (7). Consequently, the non-existence of the sign BUT in Example (7) can be treated as an instance of coordinator ellipsis (McShane 2005). Overall, the presence of the sign BUT makes distinguishing between conjunctive and adversative coordination easier as it reduces the cognitive load in deciphering the existence of the semantic opposition only on the basis of the compositional meanings (and the prosody; see Section 5) of the clauses. The sign BUT also helps the addressee to identify the clause boundary in longer sentences.

Disjunctive clausal coordination is exemplified in (10) and (11):

\section{(10) ...ME SELF GROW-UP ALREADY ' OR CULTURE CLIMATE} CHANGE...

'(I don't know if it is) me who has grown up or if the cultural atmosphere has changed...

(cfinsl2013-008-06, signer 1, coo-2)

(11) polar

h1: SPEAK OR PALM-UP

h2: $\quad$ READ

You speak it or read it?

(mocap data 1 , signer 2, example 5) 
As seen from (10) and (11), disjunctive coordination in FinSL employs the sign

497 OR in between the coordinated clauses (see Figure 2). Prosodically, as described by

498 Davidson (2013) for ASL (see Section 2.2), the disjunctive linkage in FinSL is

499 typically marked also with an emphatic change in the head or upper torso position in

500 between the two clauses (see Section 5). In (11), the distinction between the two

501 coordinands is further strengthened by the fact that the two clauses are articulated

502 with different hands.

503 Like, for example, in ASL (Davidson 2013), it is possible to express disjunctive

504 coordination asyndetically also in FinSL with distinct side-to-side head and body

505 leans. However, in practice this requires almost exaggerating the change in the

506 head/body position in between the two clauses. Consequently, in FinSL, asyndetic

507 linking in disjunctive coordination is deemed by informants to be very marked.

508 On the basis of the data analyzed, we may conclude that - conforming to the

509 general tendency of sign languages (see Section 2.2) - linking in conjunctive

510 coordination in FinSL is primarily asyndetic, whereas in adversative and in

511 disjunctive coordination FinSL prefers syndetic linking. Concerning the asyndetic

512 linking in conjunctive coordination, the question then arises of how one formally

513 distinguishes two independently produced clauses from genuine conjunctive

514 coordination. There can be no definite answer to this, and one has to acknowledge that

515 conjunctive coordination in FinSL is always ambiguous in this sense. However, some

516 cues are provided by the prosody, especially in terms of the signing speed and

517 pausing. If the signing speed is relatively fast (as in all of the examples in this

518 section), then the two clauses are very likely to form a single sentence. In contrast, if

519 there is a noticeable pause between the clauses (which is not the case in the examples

520 in this section), then the two clauses are more likely to be independent and isolated 
521 units. Moreover, continuous blending over the conjoined clauses (as in 4) may be

522 taken to be a further indicator of the coordinative reading.

523 Some diagnostics can be used to test the status of a construction as a

524 conjunctively coordinated sentence. One test is to convert the original declarative

525 sentence into a negative one (see Tang \& Lau 2012). As was stated in Section 3.3,

526 negative sentences in FinSL are constructed with the nonmanual negative operator

527 (headshake), whose scope always lasts to the end of the sentence. If, then, one wishes

528 to convert a proposition 'yes $\mathrm{A}$ and yes $\mathrm{B}$ ' into a negative 'no $\mathrm{A}$ and no B', a negative

529 operator must be applied to the first clause and it should apply continuously to the end

530 of the second clause. According to the informants, this holds true for the sentences in

531 (4)-(6). However, it must be noted that producing textual sentences in isolation and

532 altering their structure this way is considered by informants to be odd. Two reasons

533 for this are the ellipsis of thematic elements and blending, which together make the

534 sentences heavily context dependent.

535

\subsection{Features of syndetic coordination}

538 When clausal coordination is expressed syndetically (i.e. with the help of signs such

539 as ALSO, PLUS, BUT, or OR), the coordinators are positioned between the two

540 coordinand clauses. However, the question of whether the coordinands are more

541 linked to the first (i.e. A and B) or to the second clause (i.e. A and B) - that is,

542 whether the coordination is postpositive or prepositive - cannot be easily answered.

543 At first sight, it appears that both options are possible: for example, a not uncommon

544 situation is that the signer uses the clause boundary as the chance to have a short

545 hesitation break which, in turn, may either precede or follow the coordinator sign. In 
546 such cases, which of the coordinands the coordinator belongs to ultimately depends

547 on the context.

548 However, the distribution of eye blinks can be used as evidence that, in general,

549 coordinators are more linked to the latter of the two coordinands, that is, that the

550 coordination in FinSL is more prepositive than postpositive (i.e. A and B). Eye blinks

551 are generally acknowledged to be prosodic markers of constituent boundaries (e.g.

552 Wilbur 2000; Pfau \& Quer 2010), and this has been attested also for FinSL (Jantunen

553 2007). In coordinated clauses, eye blinks - if present - tend to occur before any

554 coordinator sign, that is, right after the end of the preceding clause. That this is the

555 fact is acknowledged in the annotation of all the examples in the present paper (see

556 also Figure 4 in Section 5).

557 The signs ALSO and BUT can also be found at the beginning of

558 sentences/clauses. This use of the signs should not be confused with the prepositive

559 type of "and A B" coordination. Rather, in such cases, these signs function as turn-

560 initial utterance particles that may connect together bits of discourse over long

561 distances (Hakulinen et al. 2004). This use of the sign BUT is demonstrated in (12).

562

563

(12) BUT TRAINING OWN WRESTLE ALWAYS ME SELF GO

564

565

'But to my wrestling training I always go alone.'

566

567 The sign OR can also occur sentence/clause-initially. However, in such cases

568 there is a semantic restriction that the sentence must be in the interrogative. The use of 569 the sign PLUS at the beginning of sentences is highly marked. 
571 Section 2.2). In fact, no traditional forms of bi- or multisyndetic coordination seem to 572 exist in FinSL (cf., however, the list buoy construction introduced in Section 2.2, and 573 the following discussion).

\subsection{Multiple coordination}

576

577 In FinSL, multiple clausal coordination is primarily asyndetic. Even the final 578 coordinated clause is linked to the list asyndetically, contrary to the typical cross-

579 linguistic strategy (Velupillai 2012). Examples in the data of the multiple conjunctive 580 coordination of FinSL clauses are given in (13)-(14).

(13) h1: CL-A-"climbing-right-paw"-2 ' CL-V-"jump"-2 ' ESCAPE h2: CL-A-"climbing-left-paw"-2 CL-S-"edge"-2 ESCAPE 'Gently the frog climbed up the wall, (the frog) jumped down from the edge of the jar, and (the frog) escaped.

591

However, as was the case with bi-coordinand conjunctive coordination, also multiple conjunctive coordination can be expressed with a syndetic strategy. In such

594 cases the primary coordinators are, again, the signs ALSO and PLUS. The coordinator 
595 appears before the final clause, as is the cross-linguistic tendency. Each of the

596 coordinated items typically forms an independent prosodic unit, the possible

597 coordinand being, on the basis of the distribution of eye blinks, for example, part of

598 the final item.

599 In multiple adversative and disjunctive coordination, the syndetic strategy with

600 the signs BUT and OR is preferred. The location of these signs in the coordinate

601 string is determined by what the signer wishes to say; that is, they can occur on any

602 boundary between the coordinated clauses.

603 No instances of the list buoy construction, introduced in Section 2.2 as a

604 strategy to express multiple coordination, were found in the data. However, according

605 to the native FinSL informants, the list buoy can be used to conjunctively coordinate

606 (typically) multiple units such as clauses also in FinSL (see also Section 6).

607

6085 Prosody of clausal coordination in FinSL

609

610 Section 4 described how there is a prosodic break in between the coordinated clauses.

611 However, it was implicitly stated that this prosodic break is different in different types

612 of coordination. This section discusses these differences in more detail.

613

\section{$614 \quad 5.1$ Disjunctive and adversative coordination}

615

616 Let us begin by briefly looking at disjunctive coordination. In general, as stated in

617 Section 4, disjunctive coordination in FinSL is strongly syndetic, that is, FinSL

618 employs the sign OR in between the coordinated clauses. In addition, the disjunctive

619 linkage in FinSL is marked with an emphatic change in the upper-body position in 
620 between the two clauses. This change is demonstrated in Figure 3, with video frames

621 showing the clausal juncture of Example (11) in Section 4.1.

622

623 \{Figure 3 approximately here

624

625

Figure 3 shows how the signer swings her upper body from right to left during the production of the coordinator OR to further mark the semantic contrast between the two disjunctively coordinated clauses (as stated in Section 4.1, the two clauses are separated in the example also by the fact that they are articulated with different hands). The figure also shows how the signer pushes her head forward during the sentence. However, although this head thrust undoubtedly makes the contrast and

631 prosodic break between the clauses even more emphatic, it is not in itself a property

632 of coordination. Instead, together with the raising of the eyebrows it forms the

633 nonmanual polar interrogative operator in FinSL (see Section 3.2; see also Puupponen

634 et al. submitted). Note also that the two positions of space in which the upper body of

635 the signer is located in the example do not have any meaning; in other words, the 636 space is not used here for any reference-tracking purpose (see Section 3.3).

637 The data shows that, in disjunctive coordination, prosody manifested by upper-

638 body behavior is used together with the manual coordinator sign (see Davidson 2013

639 for ASL). This is not what we typically see in the instances of unmarked adversative 640 coordination with the coordinator sign BUT. Consider the frames in Figure 4,

641 demonstrating the clausal juncture of Example (9) in Section 4.1. 
648 blink alone. However, the data shows that the sign BUT can be accompanied with a

649 head movement. If present, this movement is typically either a head pull or a head

650 thrust, that is, either a backwards or forwards directed movement of the head (for head

651 movements in FinSL, see Puupponen et al. submitted).

652 Tang and Lau (2012) have argued that in cases where manual markers of

653 coordination are absent, the role of nonmanual prosody is increased (see Section 2.2).

654 The present data on disjunctive and adversative coordination - both of which can also

655 be expressed asyndetically in FinSL - supports this argument. When disjunctive

656 coordination, it was stated in Section 4.1, is expressed without the sign OR, the side-

657 to-side body movement in between the clauses becomes nearly exaggerated (and such

658 an asyndetic form of disjunctive coordination is, consequently, very marked). A

659 similar effect is also seen in adversative coordination in which the coordinator BUT

660 has been omitted. Consider the example in Figure 5.

661

$662 \quad\{$ Figure 5 approximately here

663

664 The two frames in Figure 5 show the clausal juncture of Example (7) in Section

665 4.1. In the example, the adversative coordinator BUT is not present and we can

666 observe more nonmanual activity than we typically find in coordination manifested

667 syndetically with the sign BUT (e.g. in Figure 4). In particular, in Figure 5, right after

668 the clausal juncture, the upper body of the signer leans to the left and, simultaneously,

669 the head is thrust forward. Consequently, the whole structure resembles very much 
670

671

672

673

674

675

676

677

678

679

680

681

682

683

684

685

686

687

691

692

693

694

asyndetic forms of disjunctive or conjunctive coordination, the semantics together with the prosody being the only way to determine the type of coordination.

\subsection{Conjunctive coordination}

The previous section (5.1) showed that asyndetic (marked) forms of disjunctive and adversative coordination in FinSL typically employ changes in the positions and movements of the whole upper body to mark the boundary of the two coordinated clauses. In general, the same strategy can also be used in conjunctive coordination, which in the unmarked case is asyndetic in FinSL (see Secion 4.1). However, in conjunctive coordination the data indicates that the head has a more decisive role than the torso in marking the prosodic break. In other words, in conjunctive coordination it is not so much changes in the positions of the whole body that mark the prosodic break between the coordinated clauses as changes in the positions of the head (the head, of course, is part of the upper body, which makes distinguishing between head and whole body behavior sometimes very difficult). Moreover, the behavior of the head also contributes to drawing the contours of coordinand clauses, that is, in joining the units forming the clauses prosodically together.

To understand the prosodic role of the head in conjunctive clausal coordination, let us look at the data presented in Figure 6. Basically, Figure 6 is a screenshot from the ELAN annotation tool used in the annotation of the present data and it presents Example (4) in Section 4.1. However, in addition to the glosses and sentence-level translation, the screenshot also includes visualized head movement data produced with SLMotion (see Section 1.2). In Figure 6, the head movement data is represented in four panels. The uppermost panel shows the amount of horizontal (x-axis) motion 
695 of the centroid of the signer's head, tracked from the video. The three panels below

696 that show the motion of the head with respect to the yaw angles(the turning

697 movements of the head), pitch angles (the nodding movements of the head), and roll

698 angles (the tilting movements of the head), respectively. The vertical bar (with the

699 croshair) marks the area of the clausal juncture.

700

$701 \quad$ \{Figure 6 approximately here

702

703

In general, the head movement data in Figure 6 demonstrates how, in the

704 production of the example, the head moves a lot. This is partly because of the blend of

705 the signer into the boy during the sentence. However, from the point of view of

706 coordination, the behavior of the head in the dimension captured by the roll descriptor

707 (the lowest panel) is more systematic than in the others. In practice, the roll value

708 describes the sideways, tilting-like movements of the head. In Figure 6 we see that the

709 clausal juncture is associated with the lowest roll value, the descriptor thus forming a

710 valley-shape over the juncture. In practice, in terms of the roll angle, the data tells us

711 that the head position at the moment of the juncture is close to neutral whereas during

712 the production of both coordinand clauses the head is tilted to the signer's right

713 (indicated by the increasing roll value in the positive direction). The descriptor in the

714 uppermost panel, showing the sideways movement of the centroid of the head (along

715 the $\mathrm{x}$-axis), agrees with this analysis.

716 The tendency of particularly sideways tilt-like movements of the head to draw

717 the contours of the clauses in conjunctive coordination is a characteristic of all the

718 FinSL data analyzed (nonmanual prosody is known to tie syntactic units together, and

719 hence to add cohesion, also more generally; e.g. Pfau \& Quer 2010). To strengthen 
this point further, Figure 7 presents another example of the phenomenon; Figure 7

721 presents Example (5) in Section 4.1. Note that this time the figure shows only the 722 yaw, pitch, and roll desciptors (cf. Figure 6).

723

$724 \quad$ Figure 7 approximately here

725

In Figure 7, the roll angle descriptor (bottom panel) again captures the fact that

727 the head tilts to the right and returns to its neutral position during the production of 728 both coordinands, and that the clausal juncture is associated with the moment the first 729 tilt-like movement ends and the second one begins. Moreover, this example shows 730 that the clausal juncture is associated also with a nod-like movement of the head (the 731 middle panel showing the pitch angle descriptor), and that this nod-like movement 732 also participates in drawing the contour of the clauses. Here the nod-like movement 733 functions to emphasize the forward-directed manual movement of the verbal predicate 734 HOME+GO comprising the first clause (cf. Puupponen et al. submitted). In general, 735 however, unlike what has been argued to be the case with ASL and HKSL (e.g. Tang

$736 \&$ Lau; see Section 2.2), the present FinSL data with its human-made annotations

737 does not normally include head nods in inter-sentence clausal junctures (in any of the 738 three semantic types of coordination). Instead, nods tend to occur at sentence 739 boundaries.

\section{6. Sign language-specific forms of clausal coordination}

743 As was mentioned in Section 1.1, sign languages are different from spoken languages

744 in terms of their physical manifestation, and this is a cause for various modality 
745 differences between the two types of languages. In terms of coordination, Section 2.2

746 already presented the list buoy construction as one type of sign language-specific

747 form of prepositive and multisyndetic coordination strategy (see Velupillai 2012), and

748 Section 4.3 stated that the list buoy structure can be used as a mechanism to

749 coordinate multiple clauses also in FinSL. On the strength of the present FinSL data,

750 this section now presents two more structural means by which sign languages can

751 express clausal coordination. In general, we consider these means to be sign language-

752 specific in the sense that they employ natural characteristics of sign languages that are

753 either not available at all in spoken languages or are only marginally used in spoken

754 languages. In practice, the strategies are enabled by the fact that signers can use two

755 manual articulators in the production of sentences, and by the pervasive iconicity of

756 sign language structure.

757 The fact that sign languages have two (primary) manual articulators (i.e. the

758 two hands) and an array of (secondary) nonmanual articulators (e.g. facial elements,

759 the head, and the torso) makes it possible to coordinate units simultaneously.

760 Consider the boldfaced parts of the following examples: "the signer looks around in a puzzled manner"

i. 'The boy and the dog sit and look around in a puzzled manner.' looks around in a puzzled manner' 
(16) h1: CL-S-"grab-branch"-2 LOOK-FOR CL-S-"hold-on-branch"-2 h2: CL-S-"grab-branch-and-hold-on-to-it"-2

'The boy grabs the branches and looks around for the frog.'

(cfins12013-008-05, signer 2, coo-8)

In (15), visualized also in Figure 8, the dominant hand (h1) represents the sitting boy and the non-dominant hand (h2) represents the sitting dog. In addition to this highly iconic manual configuration, the signer has also created a mimical blend in which she with her nonmanual upper body behavior represents the activity of the boy and the dog (i.e. she looks around in a puzzled manner). As a whole, this simultaneous construction can be analyzed either as one (two-handed) Type 3 verbal or as two simultaneously produced (one-handed) Type 3 verbals. If the former analysis is preferred, the example demonstrates the simultaneous conjunctive coordination of two NP-like elements (cf. 'the boy and the dog'). If the latter analysis is adopted, the example demonstrates the conjunctive coordination of at least two simultaneously produced clauses (cf. 'The boy sits and looks around in a puzzled manner and the dog sits and looks around in a puzzled manner'). No clear-cut solution to this analytical ambiguity is available.

\{Figure 8 approximately here

The sentence in (16) is a slightly different example of simultaneous conjunctive coordination that makes use of the existence of two hands. In this example, the signer first produces a two-handed Type 3 verbal/clause with the overall meaning 'the boy holds on to the branch of the tree'. After the completion of the sign, the non-dominant 
794 hand is retained in the configuration and position of this Type 3 verbal/clause as a so-

795 called fragment buoy while the dominant hand continues to produce the Type 1

796 verbal LOOK-FOR, corresponding to an elliptical transitive clause ('the boy looks for

797 the dog'). After this verbal/clause is produced, the dominant hand returns to the initial

798 configuration and position of the first Type 3 verbal, still present in the non-dominant

799 hand. In terms of temporality, the production of the second clause overlaps with the

800 retained and fragmented production of the first clause. Analytically, the fragment

801 buoy in the non-dominant hand functions to add cohesion to the complex sentence

802 during the production of the second clause; that is, it can be treated as a formal marker

803 that signals the interconnectedness of the two clauses. Similar examples can also be

804 found elsewhere in the data, as demonstrated in (17; see Figure 9):

805

806

807

(17) h1: CL-V-"animate-object-flies"-2 up ' LOOK-AROUND

h2: CL-B-"ground"-2 down

808

'They flew above the ground and looked down.'

(cfins12013-005-05, signer 1, coo-6)

810

$811 \quad\{$ Figure 9 approximately here

812

813 The second sign language-specific structural means to coordinate units found in

814 the FinSL data concerns the modification of the movement parameter of verbal signs.

815 Most typically, the movement parameter is modified in FinSL verbals to iconically

816 display information related to the event structure, such as the perfective aspect.

817 However, in (18), the modification is best analyzed as a means to isomorphically code

818 the temporal sequentiality of the two events ('the lamp goes on and the lamp goes 

then almost immediately closing it again. Figure 10 shows the modification with

821 frames captured from the data.

822

823

824

826

827

828

829

830

831

832

833

834

835

836

837

838

839

840

841

842

843
(18) CL-Ax-"plays with the light switch"-4u LAMP-ON/OFF

'The snowman turns the switch and is amazed to see how the light goes first on and then off.

(cfins12013-008-05, signer 1, coo-5)

\{Figure 10 approximately here

The two sign language-specific clausal coordination strategies described above (the simultaneous production of clauses, and the iconically grounded modification of the movement parameter of signs) are perhaps not the most typical ways in which FinSL (or other sign languages) express clausal coordination. However, we take them as evidence for the view that clausal coordination is, indeed, not a fixed phenomenon but rather can be seen as a gradient phenomenon (see Gast \& Diessel 2012) which, ultimately, is connected to the channel in which the language is expressed.

\section{Conclusion}

This paper has discussed the three main types of clausal coordination (conjunctive coordination, adversative coordination, and disjunctive coordination) and their typological characteristics in FinSL. It has been shown that conjunctive coordination is primarily asyndetic, although forms of syndetic conjunctive coordination also exist 
844 in FinSL. In adversative and disjunctive coordination, the syndetic strategy is

845 preferred to the asyndetic strategy, although it is a marked option in both cases.

846 Moreover, the paper has shown that syndetic coordination in FinSL (when used) is, in

847 principle, both prepositive and heavily monosyndetic. Multiple coordination of

848 clauses has also been shown to be possible.

849 Concerning the nonmanual prosody of clausal coordination, the paper has

850 discussed the various ways of marking the juncture of coordinated clauses and

851 provided evidence for the general argument that the role of nonmanual behavior

852 increases in the absence of manual markers of coordination. Moreover, on the basis

853 of, for example, computer-vision analysis of the head movements of the signer

854 appearing on the videos, the paper has suggested that nonmanual activity not only

855 marks the junctures of the coordinated clauses but also contributes to drawing their

856 prosodic contours. It is here suggested that this direct prosodic marking of the shapes

857 of syntactic constituents is an important feature of sign languages, to be taken into

858 account in future work.

859 When situated in the wider cross-linguistic context, the results of the present

860 paper indicate that, in terms of clausal coordination, FinSL behaves fairly similarly to

861 the world's spoken languages, which typically form the basis of all of the typological

862 work in linguistics. However, this is not to say that the visual-gestural channel of

863 FinSL - and of all sign languages - does not affect the manifestation of clausal

864 coordination. Some of these modality effects have also been addressed in this paper;

865 they include the simultaneous production of clauses as well as the iconic modification

866 of predicate verbals. In the end, it has been suggested that these sign language-

867 specific "effects" are caused most significantly by the existence of the "other" hand 
868 (together with other articulators) and by the pervasive iconicity of sign language

869 structure.

870 To conclude, future research will continue to investigate complex sentences in

871 FinSL. The next logical step is to focus on embedded structures, including instances

872 of asymmetric coordination, which is not dealt with in the present paper. A major sub-

873 goal in this work concerns the creation of a representative video-based research

874 corpus of FinSL. Only through such a corpus will it be possible to make the shift from

875 qualitative observations to true usage- and frequency-based generalizations in the

876 investigation of FinSL syntax. Happily, this work has begun and is already well under

877 way.

878

879 Acknowledgements

880

881 The author wishes to thank Outi Pippuri and Saija Kuronen for their annotation work

882 for the present study. The author also wishes to thank Tuija Wainio and Danny De

883 Weerdt for helpful discussion of the paper and its FinSL material. Special thanks are

884 also due to the anonymous reviewers of the journal as well as to Eleanor Underwood

885 for checking the English of the paper. The financial support of the Academy of

886 Finland under grants 269089 and 273408 is gratefully acknowledged.

887

888 
889

890

891

892

893

894

895

896

897

898

899

900

901

902

903

904

905

906

907

908

909

910

911

912

\section{References}

Ala-Sippola, Sanna (2012). Agentin ilmaiseminen suomalaisella viittomakielellä tuotetussa asiatekstissa [Expressing agent in a formal FinSL narrative]. MA thesis in General Linguistics, Department of Modern Languages, University of Helsinki.

Bickel, Balthasar (2010). Capturing particulars and universals in clause linkage: A multivariate analysis. In Isabelle Bril (Ed.), Clause linking and clause hierarchy: Syntax and pragmatics, 51-101. Philadelphia, PA (USA): John Benjamins.

Bril, Isabelle (2010). The syntax and pragmatics of clause linkage and clause hierarchy: Some new perspectives (Editor's introduction). In Isabelle Bril (Ed.), Clause linking and clause hierarchy: Syntax and pragmatics, 1-23. Philadelphia, PA (USA): John Benjamins.

Butler, Cristopher S. (2003). Structure and function: A guide to three major structural-functional theories. Part 2: From clause to discourse and beyond. Amsterdam: John Benjamins.

Crasborn, Onno \& Sloetjes, Han (2008). Enhanced ELAN functionality for sign language corpora. In the Proceedings of the 3rd Workshop on the Representation and Processing of Sign Languages: Construction and Exploitation of Sign Language Corpora, 39-43. Paris: ELRA.

Davidson, Kathryn (2013). 'And' or 'or': General use coordination in ASL. Semantics \& Pragmatics 6(4), 1-44. 
913 Gast, Volker \& Holger Diessel (2012). The typology of clause linkage: Status quo,

914 challenges, prospects. In Volker Gast \& Holger, Diessel (Eds.), Clause Linkage

915 in cross-linguistic perspective: Data-driven approaches to cross-clausal syntax,

916 1-36. Berlin: Mouton De Gruyter.

917 Dixon, Robert M. W. (1994). Ergativity. Cambridge: Cambridge University Press.

918 Dixon, Robert M. W. (2006). Complement clauses and complementation strategies in

919 typological perspective. In R. M. W. Dixon \& A. Aikhenvald (Eds.),

920 Complementation: A cross-linguistic typology, 1-48. Oxford: Oxford University

$921 \quad$ Press.

922 Dixon, Robert M. W. (2010). Basic Linguistic Theory. Vol. 1, Methodology. Oxford:

923 Oxford University Press.

924 Dixon, Robert M. W. \& Aikhenvald, Alexandra Y. (2000). Introduction. In Robert M.

925 W. Dixon \& Alexandra Y. Aikhenvald (Eds.), Changing valency: Case studies

926 in transitivity, 1-29. Cambridge: Cambridge University Press.

927 Dryer, Mathew S. (2001). What is Basic Linguistic Theory? URL: http://linguistics.

928 buffalo.edu/people/faculty/dryer/dryer/blt (accessed 14 April 2014).

929 Dryer, Mathew S. (2006). Descriptive theories, explanatory theories, and Basic

930 Linguistic Theory. In Felix K. Ameka, Alan Dench \& Nicholas Evans (Eds.),

931 Catching language: Issues in grammar writing, 207-234. Berlin: Mouton de

932 Gruyter.

933 Foley, William A. (2010). Clause linkage and nexus in Papuan languages. In Isabelle

934 Bril (Ed.), Clause linking and clause hierarchy: Syntax and pragmatics, 27-50.

935 Philadelphia, PA (USA): John Benjamins. 
936

937

938

939

940

941

942

943

944

945

946

947

948

949

950

951

952

953

954

955

956

957

958

959

960

Hakulinen, Auli, Maria Vilkuna, Riitta Korhonen, Vesa Koivisto, Tarja Riitta Heinonen \& Irja Alho (2004). ISO suomen kielioppi [BIG Finnish grammar]. Helsinki: Suomalaisen kirjallisuuden seura.

Haspelmath, Martin (2004). Coordinating constructions: An overview. In Martin Haspelmath (Ed.), Coordinating constructions, 3-39. Amsterdam: John Benjamins.

Haspelmath, Martin (2007). Coordination. In Timothy Shopen (Ed.), Language typology and syntactic description: Complex constructions. Volume 2, 1-51. Second edition. Cambridge: Cambridge University Press.

Hodge, Gabrielle \& Johnston, Trevor (2013). Patterns from a signed language corpus: Clause-level units in Auslan (Australian Sign Language). Paper presented at the Theoretical Issues in Sign Language Research Conference 11. University College London, 13 July 2013.

Hoyer, Karin (2012). Dokumentation och beskrivning som språkplanering: perspektiv från arbete med tre tecknade minoritetsspråk [Language documentation and description as language planning - Working with three signed minority languages]. Doctoral dissertation in General Linguistics. Department of Modern Languages, University of Helsinki.

Huang, Yan (2000). Anaphora: A cross-linguistic study. Oxford: Oxford University Press.

Jantunen, Tommi (2007). The equative sentence in Finnish Sign Language. Sign Language \& Linguistics 10(2), 113-143.

Jantunen, Tommi (2008). Fixed and free: order of the verbal predicate and its core arguments in declarative transitive clauses in Finnish Sign Language. SKY Journal of Linguistics 21(2008), 83-123. 
961

962

963

964

965

966

967

968

969

970

971

972

973

974

975

976

977

978

979

980

981

982

983

984

Jantunen, Tommi (2009). Tavu ja lause: tutkimuksia kahden sekventiaalisen perusyksikön olemuksesta suomalaisessa viittomakielessä [Syllable and sentence: Studies on the nature of two sequential basic units in FinSL]. Jyväskylä Studies in Humanities 117. Jyväskylä: University of Jyväskylä.

Jantunen, Tommi (2010). Suomalaisen viittomakielen pääsanaluokat [The main parts of speech in FinSL]. In Tommi Jantunen (Ed.), Näkökulmia viittomaan ja viittomistoon [Perspectives on sign and lexicon], 57-78. Jyväskylä: University of Jyväskylä.

Jantunen, Tommi (2013). Ellipsis in Finnish Sign Language. Nordic Journal of Linguistics 36(3), 303-332.

Jantunen, Tommi, Birgitta Burger, Danny De Weerdt, Irja Seilola \& Tuija Wainio (2012). Experiences collecting motion capture data on continuous signing. In the Proceedings of the 5th Workshop on the Representation and Processing of Sign Languages: Interactions Between Corpus and Lexicon, 75-82. Paris: ELRA.

Johnston, Trevor \& Schembri, Adam (2007). Australian Sign Language. An introduction to sign language linguistics. Cambridge: Cambridge University Press.

Karppa, Matti, Ville Viitaniemi, Marcos Luzardo, Jorma Laaksonen \& Tommi Jantunen (2014). SLMotion - An extensible sign language oriented video analysis tool. In the Proceedings of the 9th international conference on Language Resources and Evaluation (LREC 2014), 1886-1891. Paris: ELRA.

Liddell, Scott K. (1980). American Sign Language syntax. The Hague: Mouton Publishers. 
Liddell, Scott K. (2003). Grammar, gesture, and meaning in American Sign Language. Cambridge: Cambridge University Press.

Luzardo, Marcos, Matti Karppa, Jorma Laaksonen \& Tommi Jantunen (2013). Head pose estimation for Sign Language video. In J.-K. Kamarainen \& M. Koskela (Eds.), Image Analysis, 349-360. Springer.

McShane, Marjorie J. (2005). Theory of ellipsis. Cary, NC (USA): Oxford University Press.

Pfau, Roland \& Quer, Josep (2010). Nonmanuals: Their grammatical and prosodic roles. In Diane Brentari (Ed.), Sign Languages: A Cambridge Language Survey, 381-402. Cambridge: Cambridge University Press.

Puupponen, Anna, Tommi Jantunen, Ritva Takkinen, Outi Pippuri \& Tuija Wainio (2014). Taking non-manuality into account in collecting and analyzing Finnish Sign Language video data. In the Proceedings of the 6th Workshop on the Representation and Processing of Sign Languages: Beyond the Manual Channel [organized as a part of LREC 2014 at Reykjavik, Iceland, 31 May, 2014], 143148. Paris: ELRA.

Puupponen, Anna, Tuija Wainio, Birgitta Burger \& Tommi Jantunen (submitted). Head movements in the dimension of depth in FinSL: nods, nodding, head thrusts and head pulls. October 2014.

Reintges, Chris H. (2010). Coordination, converts and clause chaining in Coptic Egyptian: Typology and structural analysis. In Isabelle Bril (Ed.), Clause linking and clause hierarchy: Syntax and pragmatics, 203-265. Philadelphia, PA (USA): John Benjamins. 
1008 Rissanen, Terhi (1985). Rissanen, Terhi. 1985. Viittomakielen perusrakenne [The

1009 basic structure of sign language]. Publications of the Department of General

$1010 \quad$ Linguistics, University of Helsinki No. 12. Helsinki.

1011 Rissanen, Terhi (1998). The categories of nominals and verbals and their morphology

1012 in Finnish Sign Language. Licentiate Thesis in General Linguistics. The

1013 Department of Finnish and General Linguistics, University of Turku.

1014 Sandler, Wendy (2012). Visual prosody. In Roland Pfau, Markus Steinbach \& Bencie

1015 Woll (Eds.), Sign language: An international handbook, 55-76. Berlin: Mouton

1016 De Gruyter.

1017 Savolainen, Leena (2006). Interrogatives and negatives in Finnish Sign Language: An

1018 overview. In Ulrike Zeshan (Ed.), Interrogative and negative constructions in

1019 sign languages, 284-302. Nijmegen: Ishara Press.

1020 Suvi = Suvi, the Online Dictionary of FinSL. URL: http://suvi.viittomat.net (accessed

$1021 \quad 14$ April 2014).

1022 Takkinen, Ritva, Tommi Jantunen \& Outi Ahonen (forthcoming). Finnish Sign

1023 Language. In Julie Bakken Jepsen, Goedele De Clerck, Sam Lutalo Kiingi \&

1024 William B. McGregor (Eds.), Sign languages of the world. In preparation for

1025 publication with Mouton De Gruyter.

1026 Tang, Gladys \& Lau, Prudence (2012). Coordination and subordination. In Roland

1027 Pfau, Markus Steinbach \& Bencie Woll (Eds.), Sign language: An international

1028 handbook, 340-365. Berlin: Mouton De Gruyter.

1029 Van Valin, Robert D. (2005). Exploring the syntax-semantics interface. Cambridge:

$1030 \quad$ Cambridge University Press.

1031 Van Valin, Robert D. \& LaPolla, Randy J. (1997). Syntax: Structure, meaning and

1032 function. Cambridge: Cambridge University Press. 
1033 Velupillai, Viveka (2012). An introduction to linguistic typology. Amsterdam: John $1034 \quad$ Benjamins.

1035 Wilbur, Ronnie B. (2000). Phonological and prosodic layering of nonmanuals in ASL.

1036 In Karen Emmorey \& Harlan Lane (Eds.), The signs of language revisited: An

1037 anthology to honor Ursula Bellugi and Edward Klima, 215-244. Mahwah, New

$1038 \quad$ Jersey: LEA.

1039 Zeshan, Ulrike (2004). Hand, head, and face: Negative constructions in sign

$1040 \quad$ languages. Linguistic Typology 8, 1-58.

1041 


\section{Appendix 1.}

1043

1044 The coding and transcription conventions used in this paper.

1045

1046 Note that examples cited from the literature are represented in their original form and

1047 the conventions listed here do not necessarily apply to them.

1048

1049 Manual behavior in signs

1050

1051 WOMAN Signs are referred to, according to the standard convention in sign

1052 language research, as glosses, which are to be understood as rough

1053 translations of the signs' core meaning. Notation in capitals.

1054

1055

LOOK-AT A hyphen is used when a single sign is glossed with more than

1056 one (English) word.

1057

1058

$\mathrm{HOME}+\mathrm{GO}$

Consecutive signs in compound-like structures are indicated by plus signs.

1060

106

HOUSE-2

A gloss followed by a hyphenated number or numbers identifies

1062

a directional sign (i.e, that the sign is either directed to a certain

1063 location or produced at a certain location). Spatial locations are

1064 drawn from Figure 11, below, from Rissanen (1985: 18). The

1065 numbers may be followed by words (e.g., up and down) further specifying the location that is pointed at. 
$1068 \quad$ \{Figure 11 approximately here

1069

1070 INDEX-3 A non-pronoun pointing made with the index finger. As with other

1071 signs, the number indicates the relative point the sign is directed

1072 toward (see Figure 11).

1073

1074 CL-G- A CL-"letter" notation at the beginning of the gloss indicates

1075 that the corresponding sign contains a classifier handshape.

1076 Handshape symbols are based on Rissanen (1985: 68-69).

1077

1078 -"come-to-a-stop"-4-1 The end part of the gloss in Type 3 signs describing the sign's movement. The written sequence in between the quotation 1080 marks describes the overall manner of the movement. Numbers

1081 indicate the change in the location of the hand in the signing space

1082 (see Figure 11).

1083

1084 "let-it-be" Gloss-like words written in regular letters in between quotation 1085 marks represent gestures.

1086

1087

n-e-n Letters separated by hyphens refer to finger-spelled words in which 1088 every letter is expressed. 
$1091 \mathrm{~h} 1 / \mathrm{h} 2$ When needed, the activity of the two hands is represented on

1092

1093

1094

1095

1096

1097

1098

1099

1100

1101

1102

1103

1104

1105

1106

1107

1108

1109

1110

1111

1112

1113

$1114 \mathrm{~V}$

Verbal sign in predicate function. together with the symbols below.

\section{Symbols and abbreviations relating to the analysis} see below).
A change in non-manual behavior without a pause (e.g., when signaling a phrase or clause boundary).

The scope of layered non-manuals (e.g., operators, mimic behavior) is typically signaled with a line above the glossing. Alternatively, this behavior may be coded

\section{[OWN WORK-PLACE] Syntactic constituents (phrases, clauses etc.) may be} indicated within square brackets. The initial bracket may be preceded by additional grammatical information (for some of which, 
$1116 \quad \mathrm{~A} / \mathrm{S}$

1117

1118

1119

$1120 \quad \mathrm{P}$

1121

1122

1123 NP

1124

1125 TOP

1126

1127 COM
A unit referring to the single (S) or more active (A) participant (prototypically the agent) in a situation encoded by an intransitive or transitive verbal, respectively.

A unit referring to the more passive participant in the situation encoded by a transitive verbal (prototypically the patient).

Syntactic phrase in which the head is a nominal.
Topic.

Comment (clause). 


\section{FIGURES}

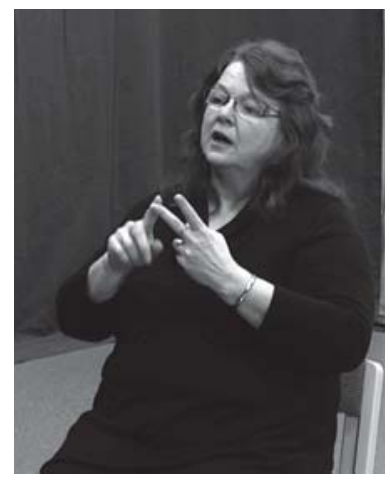

Figure 1. A FinSL list buoy meaning 'secondly'. 


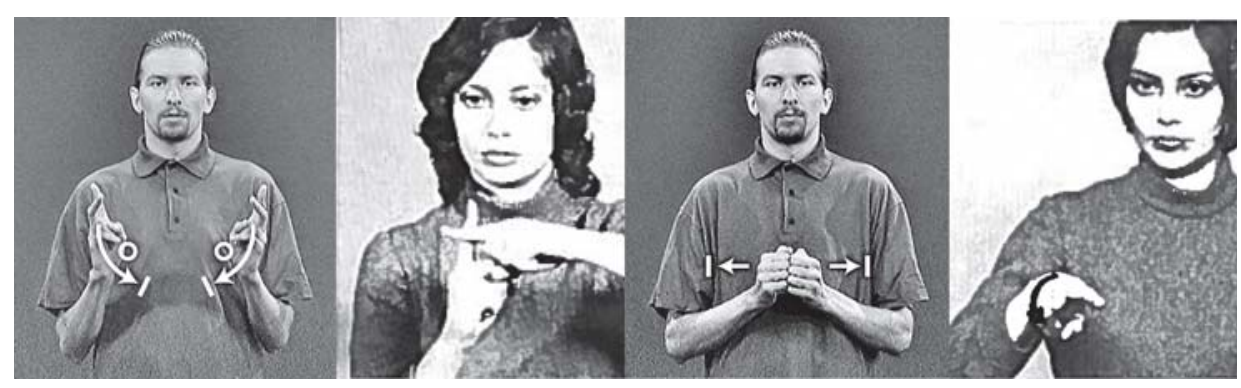

Figure 2. FinSL signs (from left to right) ALSO, PLUS, BUT, and OR. 


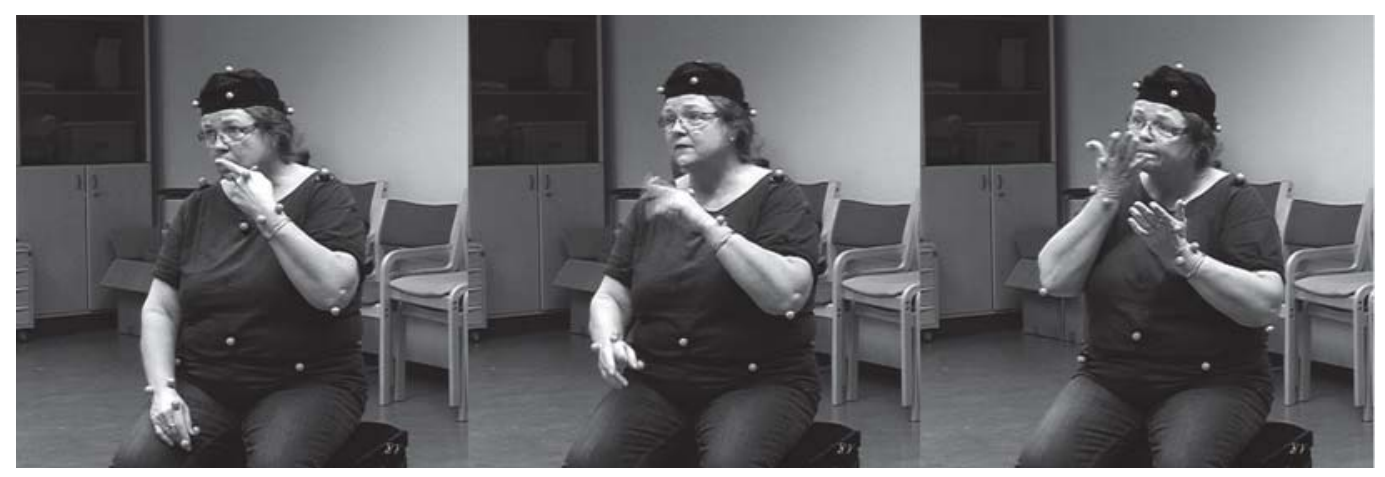

Figure 3. The articulation of FinSL signs SPEAK (left), OR (middle), and READ

(right) forming the clausal juncture of Example (11) in Section 4.1. 


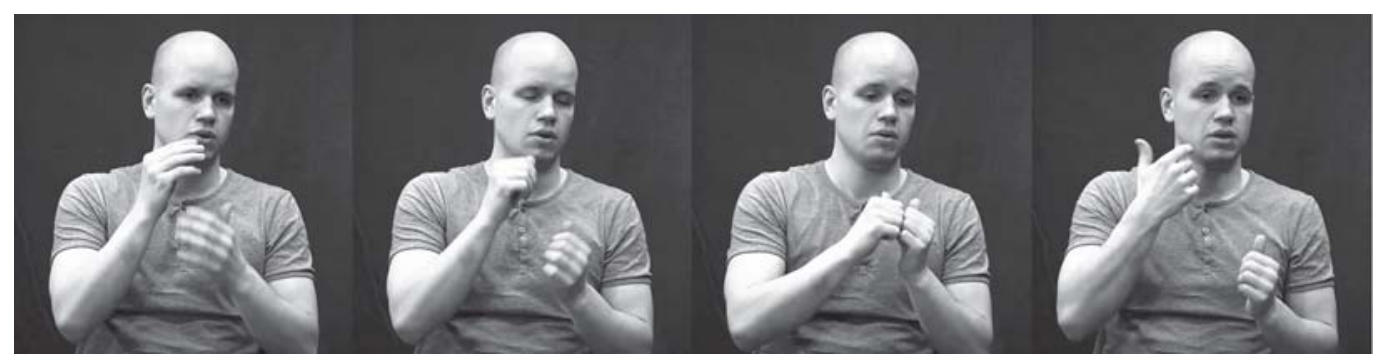

Figure 4. The articulation of the FinSL sign YES (left), eye blink and the consecutive sign BUT (the two frames in the middle, respectively), and the beginning of the sign NOW (right), all forming the clausal juncture of Example (9) in Section 4.1. 


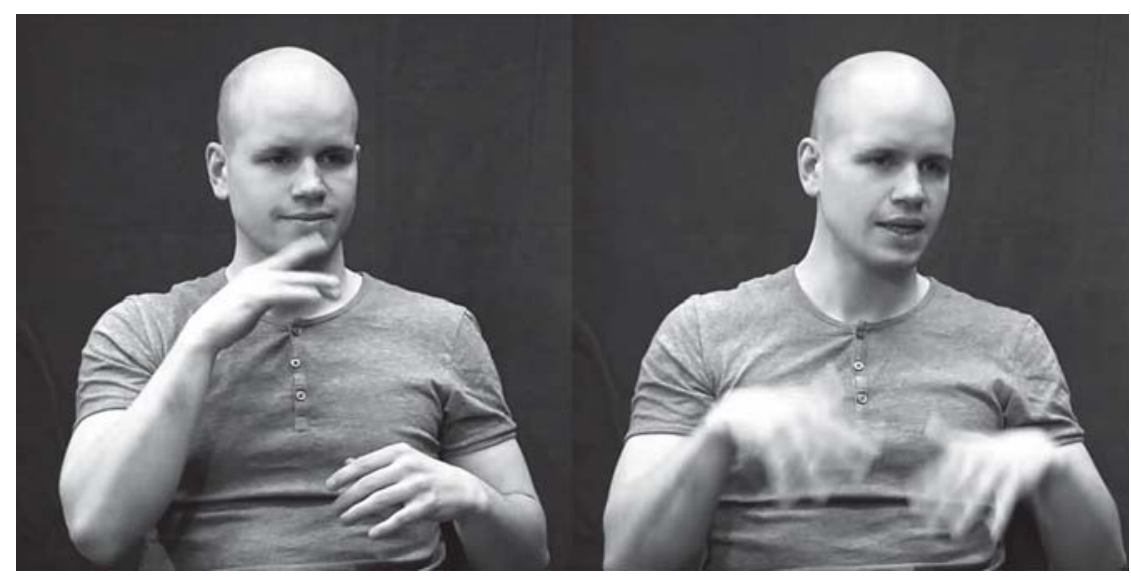

Figure 5. The articulation of the FinSL signs NICE (left) and TIRED (right) forming the clausal juncture of Example (7) in Section 4.1. 


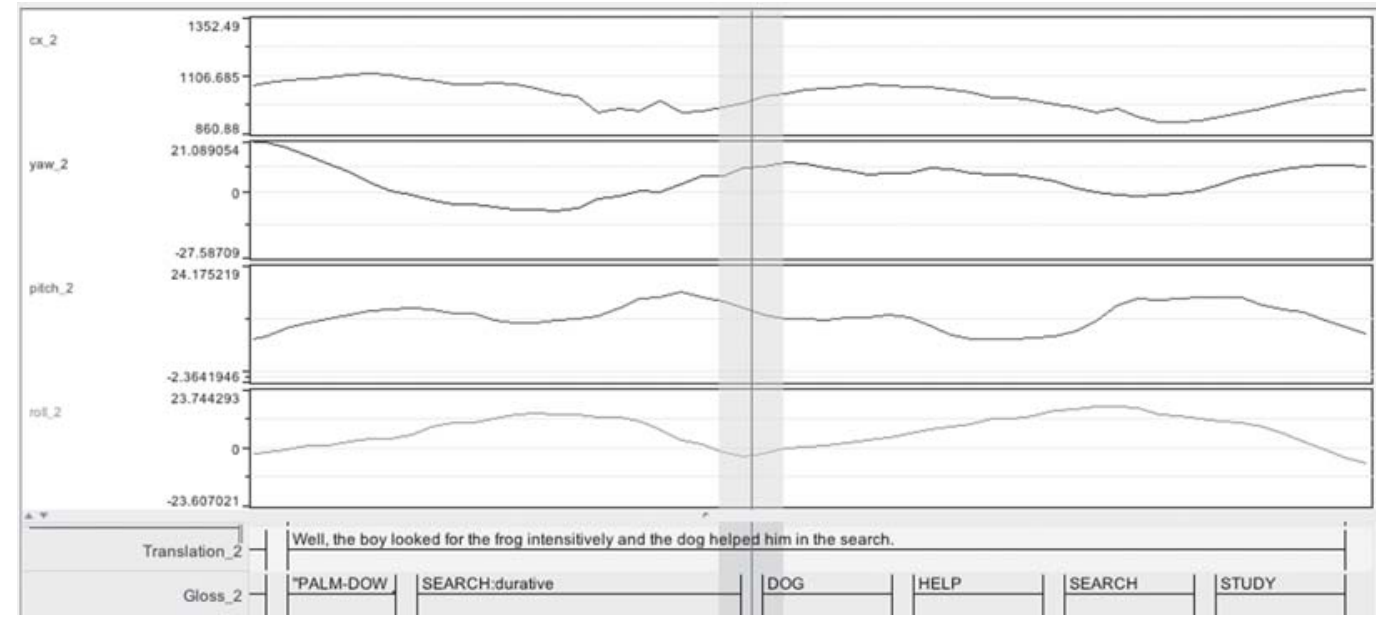

Figure 6. ELAN screenshot showing visualized head movement data for Example (4) in Section 4.1. 


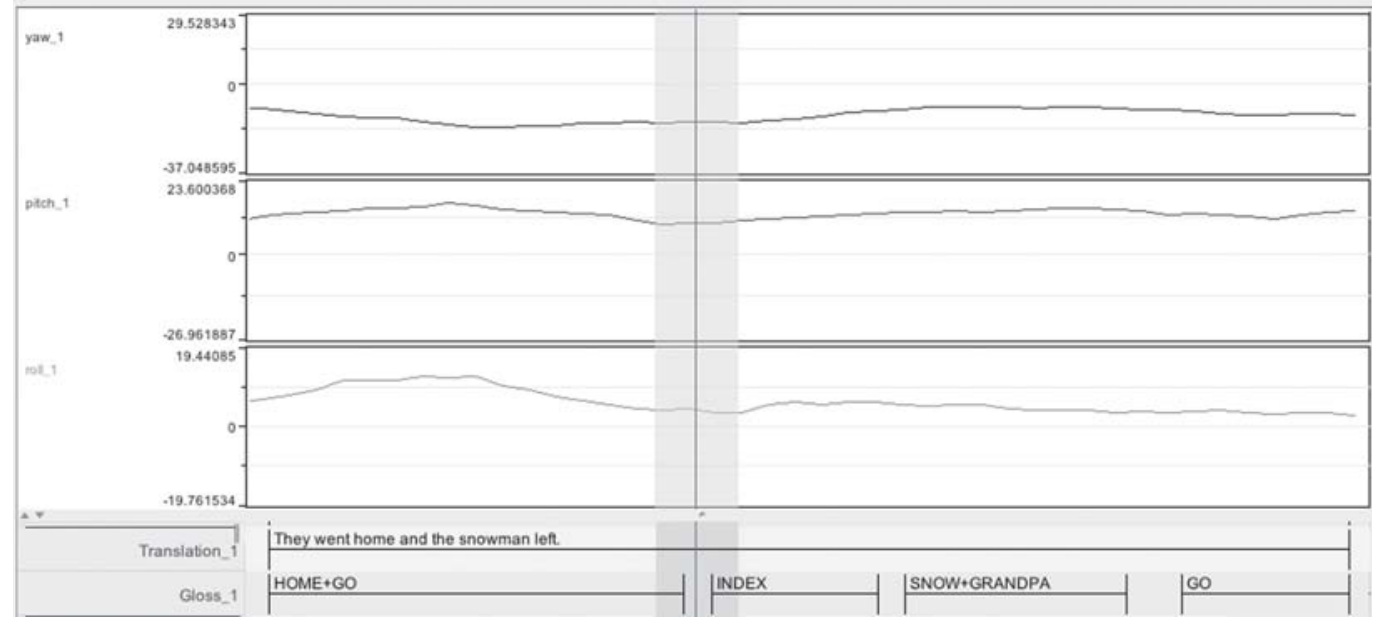

Figure 7. ELAN screenshot showing visualized head movement data for Example (5) in Section 4.1. 


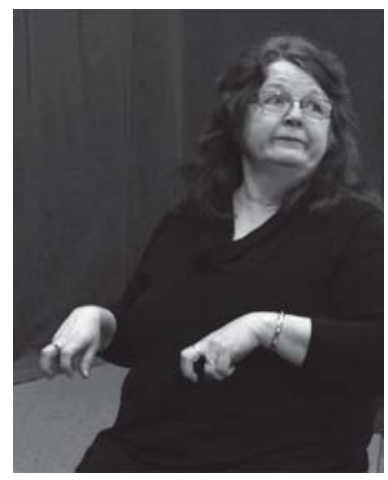

Figure 8. A frame from the data showing the simultaneous conjunctive construction in (15). 


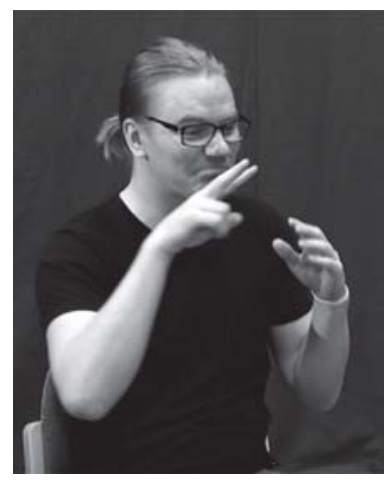

Figure 9. A frame from the data showing the production of the final clause in (17). The non-dominant hand is retained in the same position as in the first clause. 


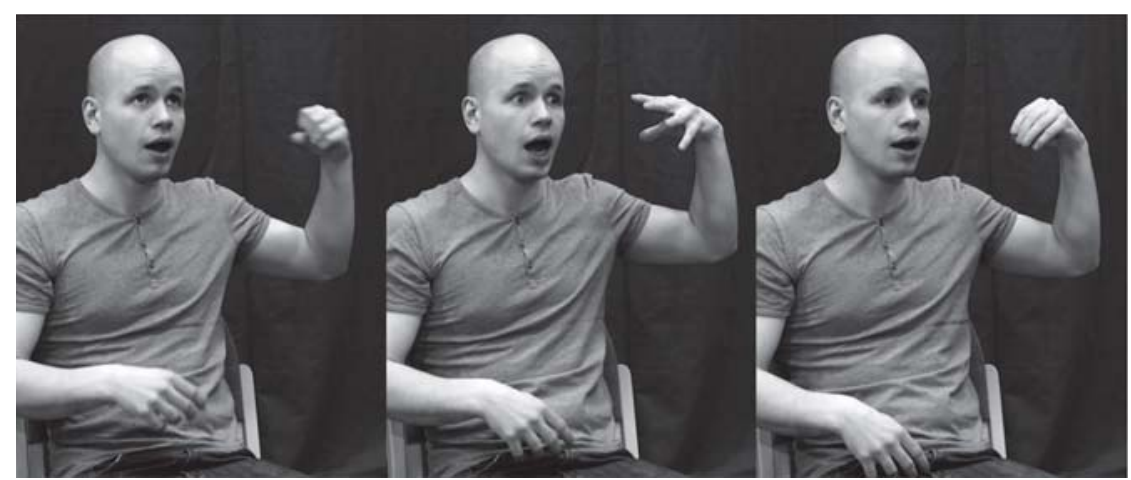

Figure 10. An example of the modification of the movement parameter of a verbal resulting in two conjunctively coordinated clauses. 


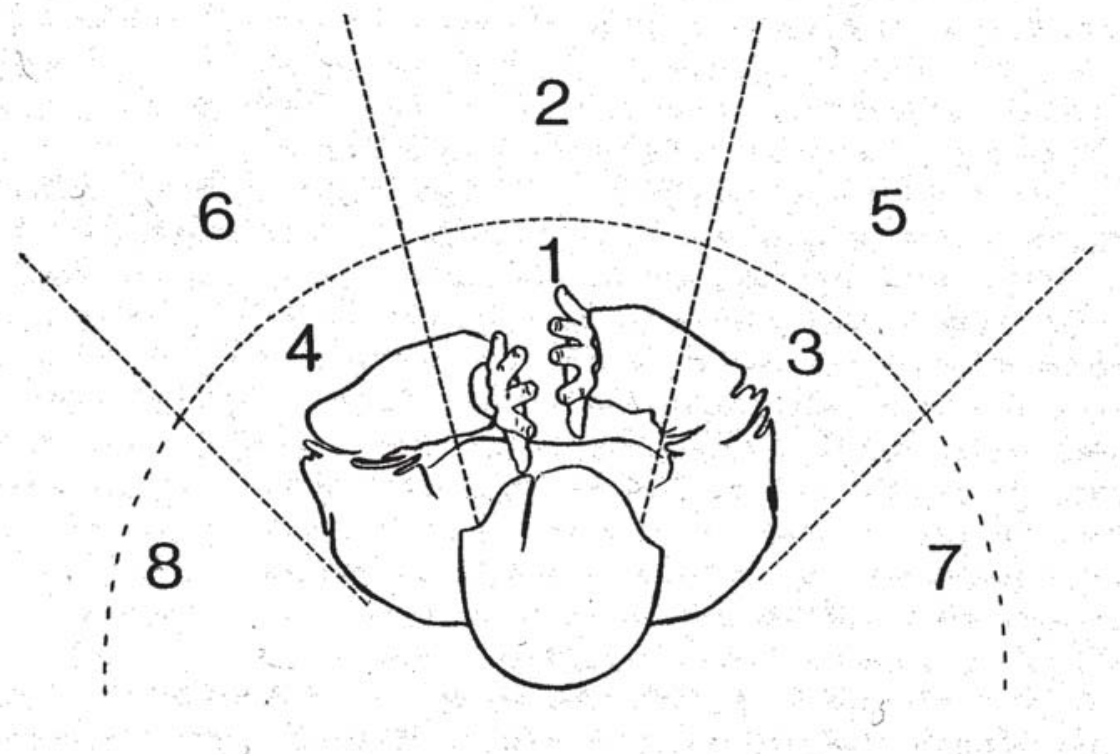

Figure 11. Locations in signing space (from Rissanen 1985: 18). 